\title{
Medicine in Focus
}

\section{Colorectal cancer: potential therapeutic benefits of vitamin $D$.}

\author{
Ana M. Jiménez-Lara \\ Department of Gene Expression Regulation, Institu to de Investigaciones Biomédicas \\ « Alberto Sols »/ Consejo Superior de Investigaciones Científicas (CSIC)/ Universidad \\ Autónoma de Madrid (UAM). C/ Arturo Duperier, 4. E-28029 MADRID, SPAIN.
}

\section{Corresponding author:}

Ana M. Jiménez-Lara

E-mail: amjimenez@,iib.uam.es

Phone: + (34) 915854454

Fax: + (34) 915854401 


\begin{abstract}
Colorectal cancer is a disease that originates from the neoplastic transformation of epithelial cells of the colon and rectum, as a result of the accumulation of genetic and epigenetic aberrations. At least four sequential genetic changes, affecting one oncogen $(K R A S)$ and three tumor-suppressor genes (APC, SMAD4 and TP53), are required for the development of colorectal cancer. Abundant experimental studies and epidemiological data, as well as several human clinical trials suggest a protective effect of vitamin D against colon carcinogenesis. Hypercalcemia, a side effect of natural vitamin $\mathrm{D}$, has currently restricted its therapeutic use; however, the development of new synthetic analogs with reduced hypercalcemic activity is promising for cancer therapy and prevention. Extensive research to elucidate the mechanisms underlying the anticancer action of vitamin $\mathrm{D}$ is being undertaken. Understand ing the complex molecular and cellular networks induced by vitamin D or its analogs will improve the use of these compounds for the prevention and treatment of colorectal cancer.
\end{abstract}

Keywords: vitamin D; vitamin D analogs; VDR; colorectal cancer; chemoprevention.

\title{
1. INTRODUCTION.
}

Colorectal cancer (CRC) is the fourth most common human malignant neoplasia worldwide, after lung, breast and prostate cancers, estimating 1000000 new cases (9.4\% of total cancer cases) and 500000 deaths annually. CRC prevalence is estimated in 2.8 million persons alive within 5 years of diagnosis, immediately after to that of breast cancer. The general incidence rates are higher in economically developed countries of North America, Australia, Eastern Asia, and Western Europe, whereas Africa, Latin America and Asia have a lower incidence. These variations in CRC 
incidence probably depend on different exposure to environmental and dietary factors. In this sense, migration studies have provided additional evidence by demonstrating that $\mathrm{CRC}$ incidence increases dramatically in populations moving from low to high-risk areas, suggesting that diets, life habits and other environmental conditions are major risk factors on CRC (Parkin et al., 2005). Accordingly, diets rich in fats and processed meat, and alcohol abuse increase the risk to suffer large bowel caner. Similarly, CRC is favored by low methionine and folate diets. Emerging evidence supports a protective effect of calcium and vitamin D in colorectal neoplasia (Lipkin et al., 1999).

\section{MOLECULAR GENETICS OF COLORECTAL CANCER.}

The molecular pathogenesis of colon cancer has been one of the most prominent research areas in the last years. The majority of the malignant colorectal tumors are sporadic, and only 5 to 10 percent are inherited as dominant autosomal. Familial Adenomatous Polyposis (FAP) and hereditary nonpolyposis Colorectal Cancer (HNPCC) are the two major forms of inherited CRC (Lynch \& de la Chapelle, 2003). $\mathrm{CRC}$ is thought to be a result of a series of genetic mutations, which parallel histopathologic and molecular changes, from normal colonic epithelium to invasive carcinoma. Interestingly, the biological properties of the resulting tumor depend primarily on the temporary sequence of mutations rather than on the accumulation of these genetic alterations.

Aberrant crypt foci (ACF) in the colorectal mucosa are considered the earliest morpho logical lesion in the development of colon neoplasia, and are currently classified as nondysplastic and dysplastic ACF. Generally, nondysplastic ACF, which harbor $K$ $R A S$ mutations, rarely lead to CRC. In contrast, dysplastic ACF contain mutations in the adenoma polyposis coli $(A P C)$ gene, and represent precursor lesions of colorectal 
adenomas and adenocarcinomas. Indeed, $A P C$ is considered the initiating "gatekeeper gene" in the majority of CRCs. The selective advantage acquired by the loss of APC function depends on the unregulated expression of the Wnt/ $\beta$-catenin/T cell factor (Tcf) target genes, basically involved in cell-cell adhesion and cell cycle control. Fifty seven percent of the dysplastic ACF contain $K-R A S$ mutations; thus, mutations in both $A P C$ and $K-R A S$ genes are relevant early molecular events in the development of CRC. Subsequent disruption of the TGF $\beta$ signaling pathway by mutations in SMAD4, SMAD2 or TGFBR2 genes, together with the altered Wnt signaling pathway, promotes a synergistic effect leading to a more malignant phenotype. Inactivation of the tumor suppressor gene TP53 is associated with adenoma-carcinoma transition in $50 \%$ of the CRC cases. Upon TP53 inactivation, cells lose cell cycle and mitotic checkpoints, accumulating mutations in other genes, which cooperate in promoting genomic instability and enhanced metastatic potential. This sequence of events resulting from APC and TP53 gene defects, account for $85 \%$ of sporadic CRC and $100 \%$ FAP patients, and is associated to chromosome instability (CIN), allelic loss and aneuploidy. Microsatellite instability (MIN) is a second type of genetic instability in CRC, and results from epigenetic silencing or mutations in the mismatch repair genes (MMR). Alterations in the $M M R$ genes occur in $15 \%$ of sporadic and in all HNPCC patients. Interestingly, these CRC cases seem to follow a distinct genetic route, which does not result in bulk genomic aberrations and aneuploidy, although $A P C$ is likely involved (Fearon \& Vogelstein, 1990; Fodde et al., 2001).

\section{VITAMIN D: POTENTIAL USE IN COLORECTAL CANCER PREVENTION AND THERAPY.}


1 $\alpha, 25$-dihydroxy vitamin $\mathrm{D}_{3}$ or calcitriol $\left[1 \alpha, 25(\mathrm{OH})_{2} \mathrm{D}_{3}\right]$, the most active molecular form of vitamin D, is synthesized from dietary-derived cholecalciferol, or from skin-derived precursors (7-dehydrocholesterol) through the action of UV sunlight. Most of the biological actions of $1 \alpha, 25(\mathrm{OH})_{2} \mathrm{D}_{3}$ are transduced by the vitamin $\mathrm{D}$ receptor (VDR), which is a member of the nuclear receptor superfamily of liganddependent transcription factors. VDR preferentially acts as a heterodimer with RXR (retinoid $\mathrm{X}$ receptor) through binding to specific genomic DNA sequences known as vitamin D response elements (VDRE), located at the regulatory regions of target genes. The interaction of $1 \alpha, 25(\mathrm{OH})_{2} \mathrm{D}_{3}$ with VDR induces allosteric changes in its ligandbinding domain and enables the recruitment of co-activators. These co-activators comprise chromatin remodeling factors, histone acetylases $(\mathrm{CBP} / \mathrm{p} 300$ and the p160 coactivators), and proteins that interact directly with the basic transcription machinery (such as the Mediator-TRAP/DRIP complex). They are able to induce chromatin decondensation, RNA polymerase II recruitment and transcriptional activation (Aranda $\&$ Pascual, 2001). Although the main physiological role of $1 \alpha, 25(\mathrm{OH})_{2} \mathrm{D}_{3}$ is the control of calcium homeostas is, however, compelling epidemiological, animal and molecular reports suggest novel activities of vitamin D.

Numerous studies have provided indirect evidence for the relationship between low vitamin D status and the risk of CRC. One of the initial reports analyzed the geographic distribution of CRC deaths in the United States (U.S.), showing that CRC mortality rates were higher in the northern regions. Additional studies evidenced a high correlation between latitude and risk of CRC in the U.S. and Europe. It appears that people living at higher latitudes, presumably synthesize less vitamin $\mathrm{D}$, and have a higher risk of dying from many common cancers including CRC. These observations closely parallel those which revealed that CRC mortality in the U.S. was higher in 
places where people were exposed to the lowest mean solar radiation, suggesting that vitamin D may play a protective role in CRC (Garland \& Garland, 1980). Moreover, many epidemiologic studies show that high vitamin D intake and high plasma levels of 25-hydroxycholecalciferol $\left[25(\mathrm{OH}) \mathrm{D}_{3}\right]$ are associated with a significant reduction of CRC incidence (Gorham et al., 2005; Grant \& Garland, 2004; Holt et al., 2002).

Experimental studies with rodent models of intestinal cancer suggest that vitamin D supply abrogates increased hyperproliferation and adenoma formation induced by stress diet (high fat, low calcium, high phosphate and low vitamin D) or carcinogen treatment (azoxymethane or 1,2-dimethylhydrazine). Treatment with vitamin $\mathrm{D}$ analogs in murine models of tumor xenografts elicited similar results. Moreover, administration of the synthetic vitamin D analogs Ro 25-6760 and EB1089 to mice with HT-29 or LoVo tumor xenografts significantly inhibited tumor growth. Mice carrying a dominant mutation in the $A P C$ gene derived from ethylnitrosourea treatment $\left(A p c^{\mathrm{min}}\right)$ or mice with targeted mutations of the APC gene $\left(A p c^{\Delta 716}, A p c^{1638}\right)$ provide a well-defined model for spontaneous CRC initiation and FAP disorders. $A p c^{1638}$ mice fed with a western-style diet (high-fat and phosphate, low in calcium and vitamin D) showed higher incidence of carcinomas and invasive tumors, demonstrating that diet modulates the incidence of carcinoma. Notably, intra-peritoneal treatment of $A p c^{\min }$ mice with vitamin $\mathrm{D}$ or a non-calcemic synthetic analog reduced tumor load by $36 \%$. Remarkably, wild type mice and rats fed with western-style diet for their entire life span will develop colon single-crypt dysplastic lesions and focal hyperplasias, indicative of tumorigenesis, which are prevented by increasing dietary calcium and vitamin D (Harris \& Go, 2004).

The previous data may suggest that VDR knockout animals should be prone to develop colon cancer. In fact, these animals exhibit high proliferating cell nuclear 
antigen activity, cyclin D1 expression, hyperproliferation and oxidative stress in the distal colon; however, they have normal colon cancer rates (Kallay et al., 2001).

\subsection{MECHANISMS OF VITAMIN D ACTION IN COLORECTAL CANCER.}

The anti-cancer activities exerted by $1 \alpha, 25(\mathrm{OH})_{2} \mathrm{D}_{3}$ and its analogs in human colon cancer cells in vitro, are mediated by proliferation inhibition and induction of differentiation and apoptosis (Lamprecht \& Lipkin, 2003). The anti-proliferative effect of vitamin D is attained by inducing G1 cell-cycle arrest, which is probably mediated by up-regulation of cell-cycle inhibitors, such as $\mathrm{p} 21^{\mathrm{WAF} 1 / \mathrm{CIP}}$ and $\mathrm{p} 27^{\mathrm{KIP}}$. Vitamin D modulates the activation of these cell cycle related genes by different mechanisms. p21 contains a VDRE in its promoter region, and therefore is susceptible of direct transcriptional control by vitamin D. However, the induction of p27 expression, devoid of VDRE, is mediated by Sp1, NF-Y transcription factors and by post-transcriptional mechanisms. Moreover, vitamin D modulates the expression of proliferation-related genes such as c-myc, c-fos and c-jun (Scaglione-Sewell et al., 2000).

Vitamin D also exerts anti-cancer effects by interfering the synthesis of growth factors and cytokines and/or modulating their signaling pathways. Normal colonic epithelial proliferation is inhibited by TGF- $\beta$ signaling. As previously discussed, disruption of TGF- $\beta$ signaling is involved in CRC malignant progression. In some models, vitamin D can restore sensitivity to TGF- $\beta$ by inducing the expression of the TGF- $\beta 1$ receptor (Chen et al., 2002).

In addition to the growth inhibitory effects, vitamin $\mathrm{D}$ induces the differentiation of colon cancer cells. In Caco- 2 and HT-29 cells, treatment with $1 \alpha, 25(\mathrm{OH})_{2} \mathrm{D}_{3}$ enhances morphological differentiation parameters, such as the number of desmosomes, intermediate filaments and microvilli length and density. Moreover, $1 \alpha, 25(\mathrm{OH})_{2} \mathrm{D}_{3}$ 
significantly increases the expression and activity of alkaline phosphatase, a marker of colonic differentiation. In SW480 colon cancer cells, the activation of VDR by $1 \alpha, 25(\mathrm{OH})_{2} \mathrm{D}_{3}$ produces dramatic changes in adherent junctions integrity, increases differentiation and reduces oncogenic cell signaling. More specifically, $1 \alpha, 25(\mathrm{OH})_{2} \mathrm{D}_{3}$ induces the expression of E-cadherin, and other adhesion proteins (occludin, zonula ocludens (ZO-1 and ZO-2), and vinculin), the translocation of $\beta$-catenin, plakoglobin, and $\mathrm{ZO}-1$ from the nucleus to the plasma membrane, and the transrepression of $\mathrm{Wnt} / \beta$ catenin/Tcf signaling (Palmer et al., 2001). The Wnt signaling pathway regulates gene expression, cell adhesion, and tissue development. Wnt ligands or mutations in key regulators of the Wnt-pathway, such as APC and $\beta$-catenin (early genetic alterations in CRC) ultimately result in the nuclear accumulation of $\beta$-catenin, where it binds to the TCF/LEF family of transcription factors, leading to the regulation of Wnt-target genes (PPAR $\delta$, Tcf-1, matrilysin, cyclin D1, and CD44). Induction of these genes has dramatic effects on cell and tissue development, and oncogenesis. Treatment with $1 \alpha, 25(\mathrm{OH})_{2} \mathrm{D}_{3}$ of colon cancer cells represses Wnt target genes, thus contributing to maintain the differentiated phenotype and growth arrest. The direct interaction of activated VDR with $\beta$-catenin in the nucleus suggests that competitive displacement of $\beta$-catenin from TCF sites may account for the repressive activity of $1 \alpha, 25(\mathrm{OH})_{2} \mathrm{D}_{3}$ (FIG.1).

The anti-cancer activity of vitamin D in colon cancer is also linked to apoptosis induction (FIG.2). In certain cells, apoptosis occurs subsequent to the induction of differentiation, as suggested by the increase in alkaline phosphatase activity. Although the underlying mechanisms remain unclear, it has been reported that the induction of apoptosis by vitamin D is associated with the up-regulation of the pro-apoptotic protein bak and the down-regulation of the anti-apoptotic protein bcl-2 (Diaz et al., 2000). The 
effects of vitamin D on the expression of other Bcl-2 family members, such as BAX and $\mathrm{BCL}-\mathrm{X}_{\mathrm{L}}$ are controversial, and are cell-type dependent.

Interestingly, it has been found that VDR is a receptor for the lithocholic acid (LCA), a secondary bile acid. Fat-rich diets contain high levels of LCA, which generates DNA damage in normal colon cells and, therefore, increases the risk to develop CRC. Activation of VDR by either LCA or vitamin D up-regulates CYP3A, SULT2A1, and MRP3 gene transcription; these proteins are involved in the detoxification of LCA in the liver and intestine. Thus, VDR, behaving as an intestinal bile acid sensor, can protect colon cells from the deleterious effect of LCA and prevent the development of CRC (Makishima et al., 2002).

\subsection{VITAMIN D ANALOGS: PROMISING THERAPEUTIC OPTIONS.}

The clinical use of $1 \alpha, 25(\mathrm{OH})_{2} \mathrm{D}_{3}$ and $1 \alpha, 25(\mathrm{OH})_{2} \mathrm{D}_{3}$ analogs for the treatment of malignancies is still in its infancy. Despite the positive results obtained with the $1 \alpha, 25(\mathrm{OH})_{2} \mathrm{D}_{3}$ analogs when analyzed with in vitro and in vivo rodents models of colon carcinogenesis, they have proved to be poorly efficient in clinical assays. A major hindrance for the translation from basic to applied research depends on the induction of hypercalcemia by with most currently available VDR ligands. In this regard, the vitamin D analog EB1089 was well tolerated in a Phase I clinical trial, including patients with advanced breast and colorectal cancers; however, clear tumor suppressive effects were not observed (Gulliford et al., 1998). Therefore, a clinical demand is to identify new VDR ligands exhibiting improved therapeutic impact, and to establish new strategies for CRC chemoprevention based on VDR ligands. The combination of vitamin D analogs with other anti-tumor agents (i.e. chemotherapeutic drugs, epigenetic drugs, etc.) may result in enhanced anti-cancer efficiency, as is the case in leukemia treatment, where 
combination therapy is successful. A big effort is being made to develop novel synthetic VDR ligands that dissociate the beneficial activities from the undesired hypercalcemic effects. For instance, VDR ligands that function in a cell- and/or gene-selective manner have been recently developed, which in vivo show a striking dissociation of biological efficiency from calcium liability (Ma et al., 2006). Interestingly, new $1 \alpha, 25(\mathrm{OH})_{2} \mathrm{D}_{3}$ analogs are able to repress AP-1 (c-Jun/c-Fos)-dependent transcription, but are impaired for the transcription of promoters containing VDRE (Castillo et al., 2006). Since the AP- 1 complex regulates the expression of genes involved in oncogenic transformation and cellular proliferation, these compounds become potential therapeutic weapons against cancer, with the additional advantage of excluding the deleterious side effects derived from the activation of VDRE-containing genes. It has been postulated that the selective activity of these VDR ligands relies on the induction of distinct conformational changes in the receptor, which may result in recruitment of different cofactors from those required for the regulation of target gene expression. In agreement with this idea, it has been shown that activated VDR binds $\beta$-catenin under conditions in which other co-activators are unable to (Shah et al., 2006). Since aberrant Wnt/ $\beta$ catenin signaling plays an important role in the development of $\mathrm{CRC}$, it would be important for the future development of vitamin D analogs to determine their ability to regulate not only VDR activity but also VDR/ $\beta$-catenin interaction and $\beta$-catenin signaling. These molecular criteria should be taken into account in order to develop novel VDR ligands with anticipated anti-cancer potential.

\section{CONCLUSIONS AND PERSPECTIVES}

The need to further explore the therapeutic and prevention potential of vitamin $\mathrm{D}$ and its non-calcemic analogs in colorectal cancer is strongly support by the abundant 
evidence provided by epidemiological, experimental and clinical studies. However, further studies are required in order to better define the signal transduction pathways and the target genes involved in the anti-cancer activity of vitamin D. Additionally, it is relevant to analyze the molecular mechanisms underlying the low sensitivity or resistance of colon cancer cells to the anti-cancer effect of vitamin D. In this context, it is necessary to identify genetic and epigenetic alterations in the key players of vitamin D signaling, accounting for vitamin D-resistance. Combined treatments of VDR ligands with drugs targeting different signaling pathways could restore vitamin D sensitivity, or increase its anti-cancer effect. Finally, the synthesis and characterization of novel VDR ligands with improved therapeutic indices and reduced side effects will become a priority research area, hopefully providing new tools in the management of colorectal cancer.

\section{ACKNOWLEDGMENTS}

I thank Professor A. Aranda and Dr. M. J. Latasa for critical reading of the manuscript, and Dr. F. M. Ruiz for his help in preparing the figures. This work was supported by the Fundación de Investigación Médica Mutua Madrileña.

\section{REFERENCES}

Aranda, A., \& Pascual, A. (2001). Nuclear hormone receptors and gene expression. Physiol Rev, 81(3), 1269-1304.

Castillo, A. I., Sanchez-Martinez, R., Jimenez-Lara, A. M., Steinmeyer, A., Zugel, U., $\&$ Aranda, A. (2006). Characterization of vitamin d receptor ligands with cellspecific and "dissociated" activity. Mol Endocrinol. (in press)

Chen, A., Davis, B. H., Sitrin, M. D., Brasitus, T. A., \& Bissonnette, M. (2002). Transforming growth factor-beta 1 signaling con tributes to caco- 2 cell growth inhibition induced by 1,25(oh)(2)d(3). Am J Physiol Gastrointest Liver Physiol, 283(4), G864-874.

Diaz, G. D., Paraskeva, C., Thomas, M. G., Binderup, L., \& Hague, A. (2000). Apoptosis is induced by the active metabolite of vitamin $\mathrm{d} 3$ and its analogue 
eb1089 in colorectal adenoma and carcinoma cells: Possible implications for prevention and therapy. Cancer Res, 60(8), 2304-2312.

Fearon, E. R., \& Vogelstein, B. (1990). A gen etic model for colorectal tumorigenes is. Cell, 61(5), 759-767.

Fodde, R., Smits, R., \& Clevers, H. (2001). Apc, signal transduction and genetic instability in colorectal cancer. Nat Rev Cancer, 1(1), 55-67.

Garland, C. F., \& Garland, F. C. (1980). Do sunlight and vitamin d reduce the likelihood of colon cancer? Int J Epidemiol, 9(3), 227-231.

Gorham, E. D., Garland, C. F., Garland, F. C., Grant, W. B., Mohr, S. B., Lipkin, M., et al. (2005). Vitamin d and prevention of colorectal cancer. J Steroid Biochem Mol Biol, 97(1-2), 179-194.

Grant, W. B., \& Garland, C. F. (2004). A critical review of studies on vitamin d in relation to colorectal cancer. Nutr Cancer, 48(2), 115-123.

Gulliford, T., English, J., Colston, K. W., Menday, P., Moller, S., \& Coombes, R. C. (1998). A phase i study of the vitamin $d$ analogue eb 1089 in patients with advanced breast and colorectal cancer. Br J Cancer, 78(1), 6-13.

Harris, D. M., \& Go, V. L. (2004). Vitamin d and colon carcinogenesis. J Nutr, 134(12 Suppl), 3463S-3471S.

Holt, P. R., Arber, N., Halmos, B., Forde, K., Kissileff, H., McGlynn, K. A., et al. (2002). Colonic epithelial cell proliferation decreases with increasing levels of serum 25-hydroxy vitamin d. Cancer Epidemiol Biomarkers Prev, 11(1), 113119.

Kallay, E., Pietschmann, P., Toyokuni, S., Bajna, E., Hahn, P., Mazzucco, K., et al. (2001). Characterization of a vitamin d receptor knockout mouse as a model of colorectal hyperproliferation and DNA damage. Carcinogenesis, 22(9), 14291435.

Lamprecht, S. A., \& Lipkin, M. (2003). Chemoprevention of colon cancer by calcium, vitamin d and folate: Molecular mechanisms. Nat Rev Cancer, 3(8), 601-614.

Lipkin, M., Reddy, B., Newmark, H., \& Lamprecht, S. A. (1999). Dietary factors in human colorectal cancer. Annu Rev Nutr, 19, 545-586.

Lynch, H. T., \& de la Chapelle, A. (2003). Hereditary colorectal cancer. $N$ Engl J Med, 348(10), 919-932.

Ma, Y., Khalifa, B., Yee, Y. K., Lu, J., Memezawa, A., Savkur, R. S., et al. (2006). Identification and characterization of noncalcemic, tissue-selective, nonsecosteroidal vitamin d receptor modu lators. J Clin Invest, 116(4), 892-904.

Makishima, M., Lu, T. T., Xie, W., Whitfield, G. K., Domoto, H., Evans, R. M., et al. (2002). Vitamin d receptor as an intestinal bile acid sensor. Science, 296(5571), 1313-1316.

Palmer, H. G., Gonzalez-Sancho, J. M., Espada, J., Berciano, M. T., Puig, I., Baulida, J., et al. (2001). Vitamin d(3) promotes the differentiation of colon carcinoma cells by the induction of e-cadherin and the inhibition of beta-catenin signaling. J Cell Biol, 154(2), 369-387.

Parkin, D. M., Bray, F., Ferlay, J., \& Pisani, P. (2005). Global cancer statistics, 2002. CA Cancer J Clin, 55(2), 74-108.

Scaglione-Sewell, B. A., Bissonnette, M., Skarosi, S., Abraham, C., \& Brasitus, T. A. (2000). A vitamin d3 analog induces a g1-phase arrest in caco-2 cells by inhibiting cdk2 and cdk6: Roles of cyclin e, p21waf1, and p27kip1. Endocrinology, 141(11), 3931-3939. 
Shah, S., Islam, M. N., Dakshanamurthy, S., Rizvi, I., Rao, M., Herrell, R., et al. (2006). The molecular basis of vitamin $\mathrm{d}$ receptor and beta-catenin crossregulation. Mol Cell, 21(6), 799-809. 


\section{FIGURE LEGENDS}

FIG.1. Molecular mechanisms of vitamin D-induced differentiation. In normal cells, the level of free $\beta$-catenin ( $\beta$-cat), which is in equilibrium with $\beta$-catenin at adherent junctions, is regulated by degradation via the proteasome. Free $\beta$-catenin is recruited to a complex with APC, axin and glycogen synthase kinase $3 \beta$ (GSK3 $\beta$ ) in the cytoplasm, and phosphorylation of $\beta$-catenin by GSK3 3 promotes its degradation by the proteasome. In SW480 colon cancer cells, as a result of APC gene mutations and low Ecadherin levels, $\beta$-catenin accumulates in the cytoplasm and is free to enter the nucleus, where it induces Tcf-dependent transcription (left). In the presence of vitamin D (right), the transcription of Tcf- target genes is repressed. Activated VDR associates with $\beta$ catenin, displacing $\beta$-catenin from Tcf-containing promoters. In this context, $\beta$-catenin might potentially function as a co-activator of certain VDR-target genes. Vitamin D reduces the nuclear levels of $\beta$-catenin by increasing its interaction with E-cadherin, which in turn is up-regulated by vitamin D treatment. $\mathrm{V}$, vitamin $\mathrm{D}$ receptor; $\mathrm{X}$, retinoid $\mathrm{X}$ receptor; VDRE, vitamin D response element; $\alpha$-cat, $\alpha$-catenin.

FIG2. Molecular basis of vitamin D-induced apoptosis. $1 \alpha, 25$-dihydroxy vitamin $\mathrm{D}_{3}$ $\left[1 \alpha, 25-(\mathrm{OH})_{2} \mathrm{D}_{3}\right]$ up-regulates the pro-apoptotic protein bak and down-regulates the anti-apoptotic protein bcl-2. Pro-apoptotic and anti-apoptotic bcl-2 family members regulate the permeability of the mitochondria outer membrane and, hence, the release of cytochrome c and other pro-apoptotic factors. Cytochrome c associates with APAF-1 and pro-caspase 9 to form the apoptosome, which triggers caspase 3 activation, inducing the cleavage of different substrates in apoptotic cells. Another bcl-2-family 
member, BID, when cleaved by caspase 8 (tBID), translocates to the mitochondria membrane and triggers the mitochondria-dependent or intrinsic death pathway. BID connects the extrinsic (death-receptor mediated cell death pathway) and the intrinsic death pathways. In prostate cancer cells, $1 \alpha, 25-(\mathrm{OH})_{2} \mathrm{D}_{3}$ induces the down-regulation of inhibitor of apoptosis proteins (IAP). APAF-1, apoptotic protease activating factor-1; BAK, Bcl-2-antagonist killer; BAX, Bcl-2-as sociated X protein; tBID, truncated BH3interacting domain death agonist; BCL-2, B-cell CLL/lymphoma-2 protein; BCL-X $\mathrm{L}_{\text {, }}$ BCL2-related protein, long isoform; FADD, FAS-associated death domain. 


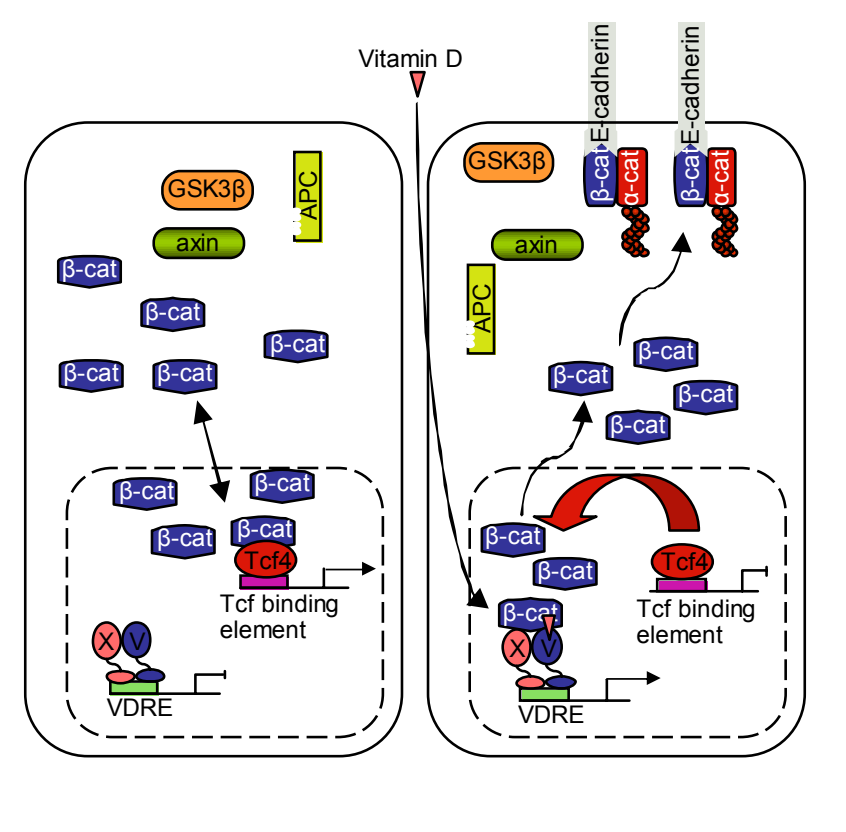

Figure-1

. 
Figure-2

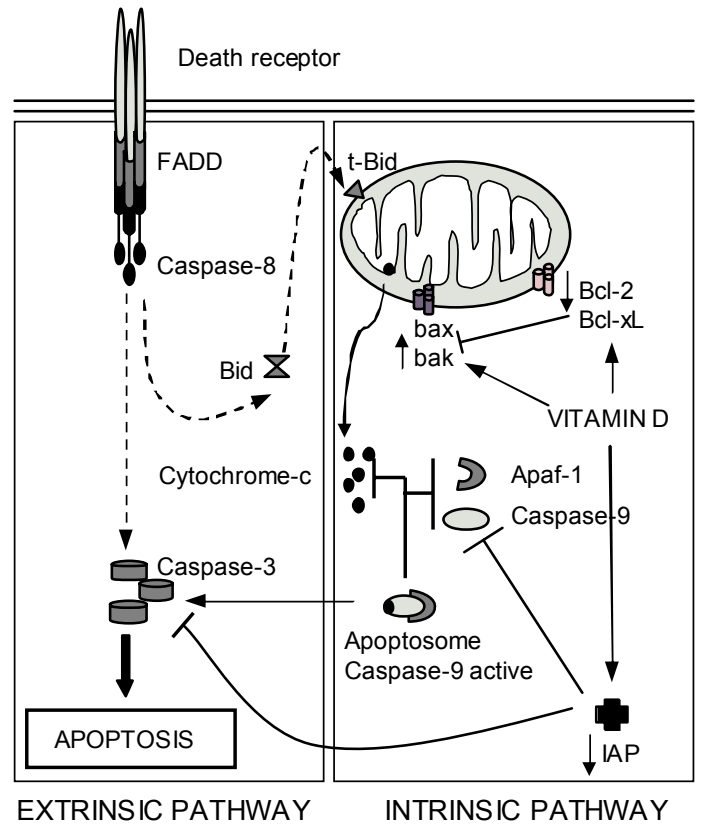

. 